\author{
Aline Macedo Carvalho Freitas ${ }^{\mathrm{a}}$ \\ (D) https://orcid.org/0000-0002-5696-9625 \\ Tânia Maria de Araújo ${ }^{\mathrm{a}}$ \\ (iD) https://orcid.org/0000-0003-2766-7799 \\ Paloma de Souza Pinho ${ }^{\mathrm{b}}$ \\ (iD) https://orcid.org/0000-0001-6402-0869 \\ Camila Carvalho Sousa ${ }^{\mathrm{a}}$ \\ (iD) https://orcid.org/0000-0002-6139-0984 \\ Paula Caroline Santos Oliveira ${ }^{a}$ \\ (iD) https://orcid.org/0000-0003-0244-4134 \\ Fernanda de Oliveira Souza \\ (iD) https://orcid.org/0000-0003-3573-9801
}

a Universidade Estadual de Feira de Santana (UEFS), Departamento de Saúde, Programa de Pós-Graduação em Saúde Coletiva. Feira de Santana, BA, Brasil.

b Universidade Federal do Recôncavo da Bahia (UFRB), Centro de Ciências da Saúde. Cruz das Almas, BA, Brasil.

Contato:

Aline Macedo Carvalho Freitas

E-mail:

amcfreitas@uefs.br

As autoras informam que este trabalho é baseado na dissertação de mestrado de Aline Macedo Carvalho Freitas, intitulada "Aspectos psicossociais do trabalho e qualidade do sono entre docentes de educação superior" e apresentada em 2018 ao Programa de Pós-Graduação em Saúde Coletiva da Universidade Estadual de Feira de Santana.

As autoras informam que dados parciais do trabalho foram apresentados em comunicação oral e publicados na modalidade de resumo nos anais do $12^{\circ}$ Congresso Brasileiro de Saúde Coletiva, realizado em 2018 no Rio de Janeiro, RJ, Brasil.

Este estudo foi financiado pela Coordenação de Aperfeiçoamento de Pessoal de Nível Superior (Capes) com a concessão de bolsa de mestrado a Aline Macedo Carvalho.

As autoras declaram que não há conflitos de interesses.

\section{Qualidade do sono e fatores associados entre docentes de educação superior}

\author{
Sleep quality and associated factors among professors
}

\section{Resumo}

Introdução: a baixa qualidade do sono pode comprometer tanto a saúde como a qualidade de vida. Objetivo: estimar a prevalência e os fatores associados à qualidade do sono ruim entre docentes de educação superior de uma universidade pública na Bahia. Métodos: estudo exploratório, transversal, realizado de novembro de 2015 a abril de 2016 com 423 docentes aleatoriamente selecionados. A qualidade do sono foi mensurada pela escala Mini-Sleep Questionnaire (MSQ). Resultados: a prevalência de qualidade do sono ruim foi de $61,3 \%$. As queixas mais frequentes foram: dificuldade de adormecer, acordar cansado, acordar com dor de cabeça e não ter tempo para dormir durante o dia. Apresentaram-se estatisticamente associadas com a qualidade do sono ruim: manter mais de um vínculo empregatício, tempo irregular e/ou insuficiente para a prática de atividades de lazer, seis horas ou menos de sono, queixas de dor musculoesquelética e de cabeça, e alta exigência psicológica com baixo controle sobre o trabalho. Conclusão: condições de trabalho que propiciam os fatores associados à elevada prevalência de qualidade do sono ruim entre docentes devem ser repensadas em prol da saúde desses trabalhadores e da importância do seu trabalho para a sociedade.

Palavras-chave: saúde do trabalhador; sono; docentes; educação superior; estudos transversais.

\begin{abstract}
Introduction: poor sleep quality can compromise both health and quality of life. Objective: to estimate the prevalence and associated factors of poor sleep quality among professors of a public university in the state of Bahia, Brazil. Methods: exploratory, cross-sectional study, conducted from November 2015 to April 2016 with 423 professors randomly selected. Sleep quality was measured using the Mini-Sleep Questionnaire (MSQ) scale. Results: the poor sleep quality prevalence was $61.3 \%$. The most frequent complaints were difficulty in falling asleep, waking up tired, waking up with a headache and not having time to sleep during the day. The following factors were statistically associated with poor sleep quality: maintaining more than one job, irregular and/or insufficient time to leisure activities, six or fewer sleep hours, complaints of musculoskeletal pain and headache, and high psychological demands with low control over work. Conclusion: the working conditions and the associated factors that provide high prevalence of poor sleep quality among professors should be rethought in favor of the workers' health and the importance of these work for society.
\end{abstract}

Keywords: occupational health; sleep; professors; higher education; cross-sectional studies. 


\section{Introdução}

O sono é um processo fisiológico necessário para a sobrevivência humana e, quando em boa qualidade, auxilia na manutenção de uma boa vigília ${ }^{1}$. A qualidade do sono, quando prejudicada, provoca, no organismo, respostas capazes de alterar o funcionamento cognitivo, comportamental, psicomotor, além de prejudicar o humor, aumentar os níveis de estresse, de cansaço, de desânimo e as queixas de dor e de outros problemas que comprometem a saúde e a qualidade de vida ${ }^{1}$.

Dentre os fatores e as situações de trabalho que contribuem para as alterações da qualidade do sono, citam-se as variáveis psicossociais, as características sociodemográficas e as experiências negativas presentes no trabalho, que agravam as respostas estressoras, elevam a liberação de glicocorticoides-GCs - hormônio regulador das reações de defesa ativadas pelo estresse e dificultam o período de recuperação e descanso dos trabalhadores antes e após as jornadas de trabalho ${ }^{2-5}$. Na educação superior, as múltiplas demandas acadêmicas compostas por carga horária elevada, atividades excessivas e pressões por aumento de produtividade, de desempenho e de competitividade, entre outras condições, desencadeiam alterações na situação de saúde $\mathrm{e}$, consequentemente, na qualidade e quantidade do sono $^{2,6}$ dos docentes atuantes na educação superior.

$\mathrm{O}$ aumento das demandas, exigências e responsabilidades impulsionam, entre os docentes de educação superior, a necessidade de criar novas habilidades sociais, emocionais e competências pedagógicas que impactam em diferentes dimensões da vida ${ }^{7}$; é possível afirmar que o sono é uma dessas dimensões afetadas ${ }^{2,8}$. Desse modo, conhecer a dinâmica que envolve o trabalho dos/as docentes universitários e sua relação com as necessidades biológicas, como o sono, é importante, uma vez que esses/as trabalhadores/as estão inseridos/as em atividades que demandam cognição, raciocínio lógico e rápido, memória e reflexão crítica.

No atual cenário, a qualidade do sono dos/as docentes da educação superior vem sendo alterada em virtude das demandas e dos arranjos no uso do tempo para as atividades do trabalho. Nesse contexto, a crescente necessidade de ampliação do tempo para o trabalho, dentro e fora do ambiente acadêmico, torna-se uma realidade, exigindo continuamente dos/as docentes universitários maior dedicação. O uso do tempo cada vez mais definido para demandas laborais e direcionado pelo esforço para cumprir as exigências acadêmicas ${ }^{9}$ é uma condição que reduz o tempo disponível para atividades pessoais, como o lazer, as horas de descanso e as de interação social e familiar.
As alterações do sono, além de causarem sérias consequências para a saúde, resultam em elevados custos financeiros para a sociedade e para as instituições de ensino, tendo em vista que o agravo dessa condição pode levar ao afastamento do/a docente das suas funções laborais.

Diante do exposto, pode-se afirmar que a baixa qualidade do sono é altamente prejudicial ao indivíduo, quer seja no contexto do trabalho, quer seja no da saúde ou, até mesmo, nos relacionamentos interpessoais. Um sono prejudicado, sem favorecimento do repouso, causa consequências que muitas vezes são imperceptíveis, porém altamente ameaçadoras para a integridade física, emocional, relacional e produtiva, tornando-se, de modo geral, um problema de saúde pública ${ }^{1,2,5}$ que merece maiores investigações e publicações, sobretudo de seu acometimento pelos docentes de educação superior.

Na medida em que os efeitos negativos da qualidade do sono interferem no desempenho do trabalho, percebe-se na literatura epidemiológica uma lacuna, haja vista que essa temática recebe pouca atenção no contexto do trabalho dos docentes universitários. Este estudo tem como objetivo estimar a prevalência e os fatores associados à qualidade do sono ruim entre docentes de educação superior de uma universidade pública na Bahia.

\section{Métodos}

Trata-se de um estudo epidemiológico de corte transversal com abordagem exploratória. O estudo é um recorte da pesquisa intitulada "Estresse ocupacional e transtornos mentais comuns entre professores universitários", desenvolvido pelo Núcleo de Epidemiologia da Universidade Estadual de Feira de Santana, Bahia, no período de novembro de 2015 a abril de 2016.

Foram elegíveis para o estudo, todos/as os/as docentes que se encontravam em efetivo exercício das suas funções, independentemente do tipo de vínculo estabelecido com a instituição. Não foram incluídos na pesquisa quem estava afastado do trabalho para aperfeiçoamento profissional ou por motivo de doença, férias, licença-prêmio ou maternidade. Foram excluídos todos aqueles que se recusaram a participar da pesquisa.

A seleção dos docentes foi feita por amostragem estratificada conforme a alocação por tipo de vínculo empregatício e departamento. Foi realizada seleção aleatória, com o uso do software Open Epi, versão 3.03a, com a utilização de procedimento numérico para identificação de participantes. 
Para o cálculo do tamanho amostral considerou-se o número total de docentes da referida universidade (931), erro amostral de 5\%, nível de confiança de $95 \%$ e prevalência esperada da qualidade do sono ruim de $46,7 \%{ }^{2}$, obtendo-se o tamanho mínimo da amostra de 271 docentes. Foram acrescidos $20 \%$ em função de possíveis perdas, totalizando 324 docentes. Ao final da coleta dos dados, 423 docentes participaram da pesquisa. Embora o estudo não tenha sido desenhado especificamente para avaliar o sono dos/as docentes, a amostra estudada tem poder suficiente para a investigação deste estudo.

Para a coleta dos dados, utilizou-se questionário padronizado, anônimo e autoaplicável, constituído por nove blocos de questões. Na tentativa de reduzir o viés de informação, todos os pesquisadores foram treinados previamente a fim de que a padronização da coleta dos dados fosse garantida. A abordagem dos/as docentes foi realizada, prioritariamente, nos dias das reuniões dos departamentos e colegiados. Quando os/as docentes não foram encontrados nas reuniões, a segunda opção foi a busca por eles/as nas salas de aula na universidade. Para evitar perdas no estudo, foram realizadas até quatro tentativas de buscas para que os/as docentes fossem encontrados/as.

A qualidade do sono foi avaliada por meio da escala Mini-Sleep Questionnaire (MSQ) ${ }^{10}$, instrumento que avalia a qualidade do sono. O MSQ é constituído por 10 questões com respostas em escala Likert que variam de 1 (nunca) a 7 (sempre). As pontuações mais altas são caracterizadas como uma pior qualidade do sono. A soma total dos escores é dividida em quatro níveis: 10 a 24 pontos indicam sono bom; de 25 a 27 pontos, sono levemente alterado; 28 a 30 pontos, sono moderadamente alterado; e $\geq 31$, sono muito alterado ${ }^{10,11}$.

Neste estudo, os resultados do MSQ foram analisados como variável dicotômica: a qualidade do sono bom foi caracterizada pela soma das respostas que apresentaram de 10 a $\leq 27$ pontos e a qualidade do sono ruim, pela soma das respostas com pontuação de $\geq 28$ a 70 pontos.

Para a análise dos dados, inicialmente foi feita a avaliação descritiva, com base nas experiências de trabalho do modelo demanda-controle (MDC $)^{12}$. Foram analisadas as características socioeconômicas, as queixas relativas à qualidade do sono, do trabalho, os hábitos de vida, as queixas de dor e os aspectos psicossociais do trabalho.

A prevalência da qualidade do sono foi avaliada conforme essas variáveis de interesse. A exploração inicial das associações brutas foi feita por análise bivariada. Foram estimadas as prevalências, as razões de prevalências e seus respectivos intervalos de confiança de $95 \%$.
A análise de regressão logística múltipla (ARLM) foi realizada conforme as seguintes etapas: 1) seleção das variáveis a partir dos objetivos do estudo e os critérios identificados na revisão da literatura; 2) verificação dos pressupostos do modelo; 3) pré-seleção das variáveis para inclusão na análise por meio do teste de razão de verossimilhança, com adoção de nível de significância de $25 \%$ ( $\leq$ 0,25); e 4) utilização do procedimento backward na ARLM para seleção do melhor modelo. Nessa etapa, a significância adotada foi de $5 \%(\mathrm{p} \leq 0,05)^{13}$.

A análise de regressão de Poisson com variância robusta foi utilizada para obter as estimativas da razão de prevalência. A qualidade do ajuste do modelo final foi verificada por meio do teste de Hosmer-Lemeshow ${ }^{13}$ e a área sobre a curva característica de operação do receptor (ROC). A análise e processamento dos dados foram feitos nos programas estatísticos Statistical Package for the Social Sciences (SPSS), versão 23.0 e Statistics Data Analysis (Stata), versão 17.

O estudo seguiu a resolução do Conselho Nacional de Saúde 466/2012, sendo submetido e aprovado pelo Comitê de Ética em Pesquisa (CEP) sob o número do parecer 1.145.223 e CAAE 44623115.8.0000.0053. Ao aceitarem participar da pesquisa, antes de receberem os questionários, todos/ as os/as participantes leram e assinaram o Termo de Consentimento Livre e Esclarecido (TCLE). A participação dos docentes na pesquisa ocorreu de maneira voluntária, com sigilo das informações obtidas em todas as etapas.

\section{Resultados}

A Tabela 1 apresenta a análise descritiva das características sociodemográficas dos docentes de educação superior. O grupo constituiu-se com predominância do sexo feminino $(52,0 \%)$, de adultos jovens com idade entre 25 e 46 anos $(51,1 \%)$, em situação conjugal com companheiro (70,6\%), com filhos $(70,3 \%)$ e com renda mensal de mais de 10 salários mínimos (65,3\%). Quanto à distribuição de docentes por departamento, o de Saúde foi aquele com maior percentual $(28,7 \%)$, seguido pelos departamentos de Letras e Artes $(12,3 \%)$ e de Ciências Biológicas (12,1\%).

Dos 423 docentes participantes deste estudo, foram analisadas 408 respostas válidas e autorreferidas sobre a qualidade do sono; portanto, houve perda de 3,5\%. Dessas, 250 atingiram pontuações maiores que 28 na escala Mini-Sleep Questionnaire $(\mathrm{MSQ})^{10,11}$, indicando prevalência de qualidade de sono ruim de $61,3 \%$ (Tabela 2 ). 
Tabela 1 Características sociodemográficas e distribuição por departamento dos(as) docentes, participantes da pesquisa, de uma universidade pública na Bahia, Brasil, 2016

\begin{tabular}{|c|c|c|}
\hline Variáveis (n) & $n$ & $\%$ \\
\hline \multicolumn{3}{|l|}{$\operatorname{Sexo}(421)^{*}$} \\
\hline Masculino & 202 & 48,0 \\
\hline Feminino & 219 & 52,0 \\
\hline \multicolumn{3}{|l|}{ Idade $(407)^{*}$} \\
\hline 25 a 46 anos & 208 & 51,1 \\
\hline 47 a 59 anos & 199 & 48,9 \\
\hline \multicolumn{3}{|l|}{ Situação Conjugal (418)* } \\
\hline Com companheiro/a & 295 & 70,6 \\
\hline Sem companheiro/a & 123 & 29,4 \\
\hline \multicolumn{3}{|l|}{ Ter Filhos $(420)^{*}$} \\
\hline Não & 125 & 29,7 \\
\hline Sim & 295 & 70,3 \\
\hline \multicolumn{3}{|l|}{ Renda Mensal (418)* } \\
\hline Até 10 salários mínimos & 145 & 34,7 \\
\hline Mais de 10 salários mínimos & 273 & 65,3 \\
\hline \multicolumn{3}{|l|}{ Departamento $(422)^{*}$} \\
\hline Física & 13 & 3,1 \\
\hline Educação & 32 & 7,6 \\
\hline Ciências Humanas e Filosofia & 33 & 7,8 \\
\hline Tecnologia & 35 & 8,3 \\
\hline Ciências Exatas & 38 & 9,0 \\
\hline Ciências Sociais Aplicadas & 47 & 11,1 \\
\hline Ciências Biológicas & 51 & 12,1 \\
\hline Letras e Artes & 52 & 12,3 \\
\hline Saúde & 121 & 28,7 \\
\hline
\end{tabular}

*respostas válidas das variáveis, excluídas as perdas.

Tabela 2 Características da qualidade, duração e queixas de sono entre docentes de uma universidade pública na Bahia, Brasil, 2016

\begin{tabular}{lcc}
\hline Variáveis $(n)$ & \multicolumn{2}{c}{ Frequências } \\
\cline { 2 - 3 } Qualidade de sono**** $(408)^{* *}$ & 158 & 38,7 \\
Boa & 250 & 61,3 \\
Ruim & & \\
Horas de sono (418)* & 274 & 65,5 \\
De 7 a 8 horas & 144 & 34,5 \\
$\leq 6$ horas & & \\
Dificuldade de adormecer (419)* & 253 & 60,4 \\
Sim & 166 & 39,6 \\
Não & & (Continua)
\end{tabular}


Tabela 2 Continuação...

\begin{tabular}{|c|c|c|}
\hline \multirow{2}{*}{ Variáveis (n) } & \multicolumn{2}{|c|}{ Frequências } \\
\hline & $n$ & $\%$ \\
\hline \multicolumn{3}{|c|}{ Acorda de madrugada (419)* } \\
\hline Sim & 129 & 30,6 \\
\hline Não & 292 & 69,4 \\
\hline \multicolumn{3}{|c|}{ Toma remédio para dormir (420)* } \\
\hline Sim & 26 & 6,2 \\
\hline Não & 394 & 93,8 \\
\hline \multicolumn{3}{|c|}{ Dorme durante o dia (421)* } \\
\hline Sim & 340 & 80,7 \\
\hline Não & 81 & 19,3 \\
\hline \multicolumn{3}{|c|}{ Acorda cansado (417)* } \\
\hline Sim & 247 & 59,3 \\
\hline Não & 170 & 40,7 \\
\hline \multicolumn{3}{|l|}{ Ronca (417)* } \\
\hline Sim & 193 & 46,3 \\
\hline Não & 224 & 53,7 \\
\hline \multicolumn{3}{|c|}{ Acorda durante a noite $(420)^{*}$} \\
\hline Sim & 168 & 40,0 \\
\hline Não & 252 & 60,0 \\
\hline \multicolumn{3}{|c|}{ Acorda com dor de cabeça $(421)^{*}$} \\
\hline Sim & 345 & 82,0 \\
\hline Não & 76 & 18,0 \\
\hline \multicolumn{3}{|c|}{ Cansaço sem motivo aparente $(420)^{*}$} \\
\hline Sim & 152 & 36,2 \\
\hline Não & 268 & 63,8 \\
\hline \multicolumn{3}{|c|}{ Possui sono agitado $(421)^{*}$} \\
\hline Sim & 163 & 38,7 \\
\hline Não & 258 & 61,3 \\
\hline
\end{tabular}

*respostas válidas das variáveis, excluídas as perdas; ***Mini-Sleep Questionnaire (MSQ) ${ }^{10-11}$.

Dentre as características do sono analisadas, a dificuldade para adormecer $(60,4 \%)$, o acordar cansado $(59,3 \%)$ e o acordar com dor de cabeça $(82,0 \%)$ foram as mais frequentes (Tabela 2).

As maiores prevalências de qualidade do sono ruim foram observadas entre o sexo feminino $(65,0 \%)$, na faixa etária de 47 a 59 anos $(62,4 \%)$, entre quem não tinha companheiro/a (68,0\%), com filhos $(63,2 \%)$, vínculo efetivo de trabalho $(64,5 \%)$, regime de trabalho de dedicação exclusiva $(62,3 \%)$, de maior titulação - doutorado e pós-doutorado (61,5\%), com mais de 10 anos no trabalho $(64,0 \%)$ e com mais de um vínculo empregatício $(72,5 \%)$. No entanto, apenas a variável ter outro vínculo de trabalho $(\mathrm{RP}=1,39$; IC 95\%: 1,05-1,84), se mostrou estatisticamente associada a qualidade do sono ruim (Tabela 3).

Tempo irregular ou insuficiente para a prática de atividades de lazer (RP: 1,43; IC 95\%: 1,23-1,66), queixas de dor na coluna (RP: 1,26; IC 95\%: 1,06-1,50), dor musculoesquelética (RP: 1,33; IC 95\%: 1,09-1,64), dor de cabeça (RP:1,38; IC 95\%: 1,16-1,64) experiências de trabalho passivo (RP:1,31; IC 95\%: 1,01-1,72) e de alta exigência (RP:1,44; IC 95\%: 1,10-1,87) estiveram associadas à pior qualidade do sono (Tabela 4).

Na análise de associação entre a qualidade do sono segundo as variáveis de interesse avaliadas simultaneamente na análise de regressão logística múltipla (ARLM), mantiveram-se significativamente associadas à qualidade do sono ruim as variáveis: tempo irregular e/ou insuficiente para a prática de atividades de lazer, horas de sono menor ou igual a seis horas, dor musculoesquelética, dor de cabeça e a experiência de trabalho de alta exigência (altas demandas conjugada a baixo controle no trabalho). Na ARLM, considerando-se as perdas obtidas no estudo, obteve-se um quantitativo final de 316 observações (Tabela 5). 
Tabela 3 Prevalência, razão de prevalência e intervalo de confiança da qualidade do sono ruim, segundo características sociodemográficas e de trabalho entre docentes de uma universidade pública na Bahia, 2016

\begin{tabular}{|c|c|c|c|c|}
\hline \multirow{2}{*}{ Variáveis (n)* } & \multicolumn{4}{|c|}{ Qualidade do Sono Ruim } \\
\hline & $n$ & $P(\%)^{*}$ *** & $R P^{* * * * * *}$ & $(\text { IC 95\%) })^{* * * * * * * *}$ \\
\hline \multicolumn{5}{|l|}{ Sexo $(407)^{*}$} \\
\hline Masculino & 109 & 56,7 & 1,00 & --- \\
\hline Feminino & 140 & 65,0 & 1,14 & $(0,97-1.34)$ \\
\hline \multicolumn{5}{|l|}{ Idade (394)* } \\
\hline 25 a 46 anos & 120 & 58,5 & 1,00 & --- \\
\hline 47 a 59 anos & 118 & 62,4 & 1,06 & $(0,90-1,25)$ \\
\hline \multicolumn{5}{|l|}{ Situação Conjugal (404)* } \\
\hline Com companheiro (a) & 165 & 57,8 & 1,00 & -- \\
\hline Sem companheiro (a) & 81 & 68,0 & 1,17 & $(1,00-1,37)$ \\
\hline \multicolumn{5}{|l|}{ Ter Filhos $(406)^{*}$} \\
\hline Não & 69 & 56,1 & 1,00 & --- \\
\hline Sim & 179 & 63,2 & 1,12 & $(0,94-1,34)$ \\
\hline \multicolumn{5}{|l|}{ Vínculo (408)* } \\
\hline Substituto/visitante & 210 & 60,6 & 1,00 & --- \\
\hline Efetivo & 40 & 64,5 & 1,06 & $(0,86-1,30)$ \\
\hline \multicolumn{5}{|l|}{ Carga Horária (407)* } \\
\hline 40 horas ou menos & 110 & 59,7 & 1,00 & --- \\
\hline Dedicação exclusiva & 139 & 62,3 & 1,04 & $(0,89-1,22)$ \\
\hline \multicolumn{5}{|l|}{ Titulação (407)* } \\
\hline Graduação/Especialização/Mestrado & 116 & 60,7 & 1,00 & --- \\
\hline Doutorado/Pós-doutorado & 133 & 61,5 & 1,01 & $(0,87-1,18)$ \\
\hline \multicolumn{5}{|l|}{ Tempo de trabalho $(408)^{*}$} \\
\hline Até 10 anos & 113 & 58,2 & 1,00 & --- \\
\hline Mais de 10 anos & 137 & 64,0 & 1,09 & $(0,94-1,28)$ \\
\hline \multicolumn{5}{|l|}{ Outro vínculo de trabalho (122)* } \\
\hline Não & 37 & 52,0 & 1,00 & --- \\
\hline Sim & 37 & 72,5 & 1,39 & $(1,05-1,84)$ \\
\hline
\end{tabular}

Tabela 4 Prevalência, razão de prevalência e intervalo de confiança da qualidade do sono ruim, segundo as variáveis: tempo para hábitos de lazer, queixas de dor e experiências de trabalho segundo o modelo demanda-controle entre docentes de uma universidade pública na Bahia, Brasil, 2016

\begin{tabular}{|c|c|c|c|c|}
\hline \multirow{2}{*}{ Variáveis(n)* } & \multicolumn{4}{|c|}{ Qualidade do Sono Ruim } \\
\hline & $n$ & $P(\%)^{* * * *}$ & $R P^{* * * * * * * * * 6}$ & $(I C 95 \%)^{* * * * * * * * * ⿲ 二 丨 匕 ~}$ \\
\hline \multicolumn{5}{|l|}{ Tempo para o lazer (407)* } \\
\hline Sim & 209 & 58,3 & 1,00 & --- \\
\hline Não & 41 & 83,6 & 1,43 & $(1,23-1,66)$ \\
\hline \multicolumn{5}{|l|}{ Dor de Coluna (320)* } \\
\hline Não & 62 & 55,7 & 1,00 & --- \\
\hline Sim & 127 & 70,5 & 1,26 & $(1,06-1,50)$ \\
\hline \multicolumn{5}{|l|}{ Dor musculoesquelética (331)* } \\
\hline Não & 54 & 52,0 & 1,00 & --- \\
\hline Sim & 151 & 69,5 & 1,33 & $(1,09-1,64)$ \\
\hline \multicolumn{5}{|l|}{ Dor de Cabeça (321)* } \\
\hline Não & 79 & 53,7 & 1,00 & --- \\
\hline Sim & 130 & 74,2 & 1,38 & $(1,16-1,64)$ \\
\hline \multicolumn{5}{|l|}{ Modelo Demanda Controle (389)* } \\
\hline Baixa exigência & 41 & 49,4 & 1,00 & --- \\
\hline Trabalho Passivo & 60 & 65,2 & 1,31 & $(1,01-1,72)$ \\
\hline Trabalho Ativo & 74 & 57,8 & 1,17 & $(0,89-1,52)$ \\
\hline Alta exigência & 52 & 71,2 & 1,44 & $(1,10-1,87)$ \\
\hline
\end{tabular}

"respostas válidas das variáveis, excluídas as perdas; "* prevalência; "**"razão de prevalência;

********intervalo de confiança (IC) de 95\%. 
Tabela 5 Estimativa do modelo selecionado na análise de regressão logística múltipla entre docentes de uma universidade pública na Bahia, Brasil, 2016

\begin{tabular}{|c|c|c|}
\hline Variáveis & $R P^{\text {*** }}$ & (IC 95\%)****** \\
\hline \multicolumn{3}{|l|}{ Tempo para atividades de lazer } \\
\hline Sim & 1,00 & - \\
\hline Não & 1,31 & $1,10-1,56^{*}$ \\
\hline \multicolumn{3}{|l|}{ Horas de sono } \\
\hline De 7 a 8 horas & 1,00 & - \\
\hline$\leq 6$ horas & 1,22 & $1,03-1,44^{*}$ \\
\hline \multicolumn{3}{|l|}{ Dor musculoesquelética } \\
\hline Não & 1,00 & - \\
\hline Sim & 1,27 & $1,01-1,60^{*}$ \\
\hline \multicolumn{3}{|l|}{ Dor de Cabeça } \\
\hline Não & 1,00 & - \\
\hline Sim & 1,30 & $1,07-1,56^{*}$ \\
\hline \multicolumn{3}{|l|}{ Experiência de trabalho (MDC) } \\
\hline Baixa exigência no trabalho & 1,00 & - \\
\hline Trabalho passivo & 1,20 & $0,89-1,60$ \\
\hline Trabalho ativo & 1,20 & $0,90-1,61$ \\
\hline Alta exigência no trabalho & 1,34 & $1,02-1,77^{*}$ \\
\hline
\end{tabular}

"p-valor<0,05; ***azão de prevalência; ****intervalo de confiança (IC) de 95\%. Análise de regressão logística múltipla com 316 observações analisadas.

\section{Discussão}

Este estudo buscou estimar a frequência da qualidade do sono ruim entre docentes universitários, avaliando os fatores relativos as características sociodemográficas, do trabalho, hábitos de vida e saúde e os aspectos psicossociais do trabalho associados à qualidade de sono observada. A prevalência global do sono ruim foi muito elevada $(61,3 \%)$, indicando que as questões investigadas neste estudo são aspectos relevantes, que merecem especial atenção entre docentes, pois se relacionam indireta ou diretamente a diversos problemas de saúde que podem decorrer de condições de trabalhos nocivas, que devem ser repensadas no contexto estudado.

A proporção de docentes com alteração da qualidade do sono identificada neste estudo foi superior a das pesquisas conduzidas com docentes de educação do ensino médio de Poços de Caldas, $(46,7 \%)^{2}$, do ensino fundamental e médio desenvolvido em Londrina $(54,3 \%)^{8}$ e inferior à obtida no estudo com docentes de educação superior de Porto Alegre, com prevalência de $76,9 \%{ }^{14}$. Aspectos culturais e contextuais do trabalho devem ser considerados nesses locais como possíveis justificativas para as diferenças encontradas. De qualquer forma, pode-se afirmar que, independentemente do estudo, as elevadas frequências encontradas reforçam o comprometimento da qualidade do sono entre os/as docentes pesquisados/as nos referidos estudos.

Manter mais de um vínculo empregatício associou-se a maior prevalência de qualidade do sono ruim. Os docentes, quando expostos a essa condição de trabalho, tornam-se mais vulneráveis a situações que demandam maior dedicação laboral e redução do sono ${ }^{15}$. O dispêndio de maior tempo para as atividades relacionadas ao trabalho, além de prejudicar a qualidade do sono, compromete o desempenho profissional, o bem-estar físico, psicológico e mental, e os hábitos de vida, como o lazer ${ }^{16-17}$.

$\mathrm{O}$ atual contexto e arranjo do uso do tempo para as atividades de trabalho têm contribuído significativamente para alterações do sono e da saúde dos docentes da universidade investigada. Neste estudo, a indisponibilidade de tempo para a prática de atividades de lazer apresentou-se associada à qualidade do sono ruim.

Estudo conduzido com professores do ensino fundamental e médio de Londrina-PR ${ }^{8}$ identificou associação entre pior qualidade do sono e percepção negativa em relação ao tempo para lazer e para a família. Estudo com docentes de educação superior $^{18}$, observou ser praticamente impossível atender, na carga horária semanal de trabalho docente, todas as demandas do trabalho, sendo necessário o uso do 
tempo que estaria disponível para a prática de atividades de lazer e descanso para o cumprimento das demandas acadêmicas ${ }^{18}$.

Esses achados confirmaram que, no atual contexto de trabalho, o docente de educação superior possui menos tempo para a prática de lazer, para o convívio social e para o trabalho criativo, haja vista que as conversas sobre o trabalho e as trocas acadêmicas em ambientes sociais e de lazer tornaram-se ações comuns entre eles ${ }^{19}$.

A qualidade do sono ruim também se associou a duração do sono menor ou igual a seis horas. Resultados semelhantes foram identificados em estudos conduzidos com docentes do ensino médio e superior $^{20-22}$. A associação encontrada pode ser consequente da redução do sono causada pela intensificação do trabalho, pela evolução tecnológica que ampliou o uso de aparelhos eletrônicos e dissipou os limites da vida privada e do trabalho e, principalmente, pelo volume de demandas a serem atendidas no trabalho docente ${ }^{24-25}$.

Ressalta-se que a qualidade do sono, quando inferior às necessidades humanas, favorece a ocorrência de problemas que afetam o desempenho cognitivo, geram sofrimento psíquico e alteram o ritmo biológico, promovendo sérios agravos para a saúde biopsicossocial $^{26-28}$.

As queixas de dor de cabeça e de dor musculoesquelética, muito frequentes entre docentes universitários ${ }^{28}$, também se associaram a maior prevalência de qualidade do sono ruim, indicando a necessidade de maior atenção para a saúde dos docentes de educação superior, bem como reflexões sobre as consequências que essas dores podem ter no desenvolvimento das atividades na universidade ${ }^{29}$.

Uma pesquisa desenvolvida com professores da rede municipal de Salvador, na Bahia, identificou prevalências de dor musculoesquelética de 41,1\% para membros inferiores e $23,7 \%$ em membros superiores $^{30}$. Outro estudo, conduzido com docentes universitários do Noroeste do Rio Grande do Sul, encontrou prevalência de dor de cabeça de 56,5\% $\%^{29}$. Esses achados reforçam que, na experiência de trabalho docente universitário, uma maior jornada de trabalho favorece tanto a ocorrência da dor como a restrição parcial do sono, que mantem a frequência e a manutenção das queixas de dor.

A presença da dor pode gerar mudanças no padrão do sono e no estilo de vida, comprometendo a autoestima, o humor, o estado emocional, a realização de atividades diárias e o desempenho do trabalho. Os sintomas podem se manifestar como sinais de tensão, fadiga, ansiedade e distúrbios emocionais, comumente conhecidos como dor tensional, causada pela privação do sono, cansaço, estresse e outras situações que excedem a capacidade de resposta do organismo ${ }^{29-31}$.

A alta exigência no trabalho também se manteve associada à qualidade do sono ruim. Essa condição implica em alta demanda psicológica e baixo controle sobre o trabalho, consistentemente associado a diversos tipos de adoecimentos ${ }^{32}$. A literatura tem registrado evidências de associação entre os aspectos psicossociais do trabalho de alta exigência e as alterações do sono entre docentes e em outras categorias de trabalho ${ }^{20,25,33-36}$.

Os docentes, quando submetidos a alta exigência, encontram dificuldades para relaxar, acalmar os pensamentos relacionados ao trabalho e iniciar a fase de recuperação e distanciamento das tensões vivenciadas durante o dia. A vivência contínua dessa experiência gera maior excitação fisiológica e cognitiva do sistema nervoso central, o que pode promover a privação parcial e/ou o atraso do início do sono ${ }^{35-37}$.

Dessa maneira, estudos têm evidenciado que, embora possam existir entre os docentes algumas especificidades que os diferenciem, deve-se atentar, no contexto da educação superior, à existência de fatores psicossociais que produzem ou contribuem para a ocorrência de diferentes desordens no organismo; dentre elas, as alterações do sono ${ }^{38-39}$.

Os resultados deste estudo possibilitam reflexões que podem contribuir para a melhoria das condições organizacionais do trabalho, da qualidade do sono, e consequentemente, da saúde física e mental dos docentes da universidade pesquisada. Contudo, devem ser consideradas algumas limitações.

A primeira refere-se ao delineamento transversal, que impossibilita identificar a sequência temporal entre exposição e o desfecho. Ou seja, não é possível avaliar se os fatores que supostamente levaram à qualidade do sono ruim, de fato, antecederam a este evento.

A prevalência utilizada para o cálculo amostral pode ser outra limitação. Desconhecemos, até o período de investigação deste estudo, dados de qualidade do sono entre docentes universitários no país. Disto decorreu o uso da referência citada ${ }^{2}$. Ou seja, apesar de haver características distintas entre docentes de diferentes níveis e redes de ensino, há, por outro lado, algo que os identifica: o fato de serem docentes; constituindo, assim, uma base comum que torna possível alguma comparabilidade de resultados.

A mensuração da qualidade do sono ruim por meio das respostas subjetivas autorreferidas também é outro aspecto que limita a interpretação dos dados. Outro fator limitante é o viés do trabalhador sadio, pois não foram incluídos, neste estudo, docentes que se encontravam afastados por motivo de férias e/ou por doenças, sendo considerados nas análises apenas 
aqueles/as que estavam em exercício das funções laborais. É possível que docentes com problemas do sono, que produziram adoecimento mais grave, possam ter sido excluídos/as do estudo. Por fim, o viés de memória também precisa ser considerado, visto que houve entre os participantes a necessidade do resgate de informações de experiências anteriormente vivenciadas.

Como ponto positivo deste estudo, destaca-se a importância da temática ainda pouco explorada na área da saúde do trabalhador e o fato da pesquisa ter sido conduzida em uma amostra representativa de docentes universitários de uma instituição pública.

\section{Conclusão}

Neste estudo, a dificuldade de adormecer, o acordar cansado e com dor de cabeça são as queixas de sono mais frequentes entre os docentes estudados/as. A alta proporção de qualidade do sono ruim entre os docentes também é bastante preocupante. Os resultados evidenciam que a ausência e/ou irregularidade da prática de lazer, o tempo de sono menor ou igual a seis horas, as queixas de dor musculoesquelética e de dor de cabeça, e a experiência de alta exigência no trabalho são fatores associados a maior prevalência da qualidade do sono ruim entre os docentes participantes. Assim sendo, são necessárias ações de promoção de saúde que contribuam para uma melhor qualidade do sono. Essas ações devem considerar, especialmente, o reordenamento das características e do contexto organizacional do trabalho docente.

Com isso, entende-se que a exploração da temática deste estudo revela-se de grande importância para os docentes de educação superior, bem como contribui positivamente com a saúde coletiva, com destaque para o campo da saúde do trabalhador. Além disso, novos estudos neste tema poderiam beneficiar toda a sociedade, em razão da importância do trabalho desenvolvido nas universidades públicas e, portanto, da necessidade de que sejam vivenciadas, nesse ambiente laboral, boas condições de trabalho, de vida e de saúde, que possam gerar bem-estar generalizado.

\section{Contribuições de autoria}

Freitas AMC, Araújo TM, Pinho PS, Sousa CC, Oliveira PCS e Souza FO tiveram contribuição substancial na concepção do estudo, no levantamento, na análise e interpretação dos dados, na elaboração, nas revisões críticas do manuscrito e na aprovação da versão final publicada e assumem responsabilidade pública integral pelo trabalho realizado e o conteúdo aqui publicado.

\section{Referências}

1. Kim LJ, Tufik S, Andersen ML. Neurofisiologia do Sono. In: Haddad FLM, Gregório LC, editores. Manual do residente: medicina do sono. Barueri: Manole; 2017.

2. Valle LER, Reimão R, Malvezzi S. Reflexões sobre psicopedagogia, estresse e distúrbios do sono do professor. Rev Psicopedag. 2011;28(87):237-45.

3. Karasek RA. Job demands, job decision latitude and mental strain: Implications for job redesign. Adm Sci Q. 1979;24(2):285-308.

4. Munck A, Guyre PM, Holbrook NJ. Physiological functions of glucocorticoids in stress and their relation to pharmacological actions. Endocr Rev. 1984;5(1):25-44.

5. Palma BD, Tiba PA, Machado RB, Tufik S, Suchecki S. Repercussões imunológicas dos distúrbios do sono: o eixo hipotálamo-pituitária-adrenal como fator modulador. Rev Bras Psiquiatr. 2007;29(Suppl 1):33-8.

6. Cortez PA, Souza MVR, Amaral LO, Silva LCA. A saúde docente no trabalho: apontamentos a partir da literatura recente. Cad. Saude Colet. 2017;25(1):113-22.

7. Santos DAS, Azevedo CA, Araújo TM, Soares JFS. Reflexões sobre a saúde docente no contexto de mercantilização do ensino superior. Rev Docencia Ens Sup. 2016;6(1):159-86.

8. Meier DAP. Qualidade do sono entre professores e fatores associados [tese]. Londrina: Universidade Estadual de Londrina; 2016.

9. Vidal A, Oliveira S, Rotenberg L. A autoconfrontação com o próprio tempo como perspectiva analítica no estudo das relações entre o tempo e a saúde. Cien Saude Colet. 2018;23(7):2393-402.

10. Zomer J, Peled R, Rubin A, Lavie P. Mini-sleep questionnaire (MSQ) for screening large populations for EDS complaints. In: Koella W, Ruether E, Schulz H, editores. Sleep'84. Proceedings of the $7^{\text {th }}$ European Congress on Sleep Research; 1984 Sep 3-7; Munich, Germany. Stuttgart: Gustav Fischer; 1985. 
11. Gorenstein C, Tavares S, Alóe F. Sleep self-evaluation questionnaires. São Paulo: Lemos; 2000.

12. Araújo TM, Karasek RA. Validity and reliability of the job content questionnaire in formal and informal jobs in Brazil. Scand J Work Environ Health. 2008;34(6):52-9.

13. Hosmer D, Lemeshow S. Applied logistic regression. 2nd ed. New York: John Wiley \& Sons; 2000.

14. Ligabue R. Prevalência de alterações de sono e estresse em docentes do ensino superior de uma instituição de ensino privada em Porto Alegre [dissertação]. Canoas: Centro Universitário La Salle; 2017.

15. Santos-Silva R, Bittencourt LRA, Pires MLN, Mello MT, Taddei JA., Benedito-Silva AA, et al. Increasing trends of sleep complaints in the city of Sao Paulo, Brazil. Sleep Med. 2010;11(6):520-4.

16. Lopes MCR, Mancebo D. Trabalho docente: compressão temporal, flexibilidade e prazer? Rev Educ Publica. 2004;24(13):138-52.

17. Penteado RZ, Pereira IMTB. Qualidade de vida e saúde vocal de professores. Rev Saude Publica. 2007;41(2):236-43.

18. Lemos DVS. Alienação no trabalho docente: o professor no centro das contradições [tese]. Salvador: Universidade Federal da Bahia; 2007.

19. Carlotto MS, Câmara SG. Preditores da Síndrome de Burnout em professores. Psicol Esc Educ. 2007;11(1):101-10.

20. Vedovato TG, Monteiro MI. Perfil sociodemográfico e condições de saúde e trabalho dos professores de nove escolas estaduais paulistas. Rev Esc Enferm USP.2008;42(2):290-7.

21. Souza JC. Caracterização do ciclo sono/vigília de professores do ensino médio em Natal/RN [dissertação]. Natal: Universidade Federal do Rio Grande do Norte; 2010.

22. Silva KN, Dutra FCMS. Fatores psicossociais do trabalho e dor crônica: análise em duas escolas da rede municipal de educação em Serrana. Revista Dor. 2016;17(3):164-70.

23. Mancebo D. Trabalho docente: subjetividade, sobreimplicação e prazer. Psicol Reflex Crit. 2007;20(1):74-80.

24. Cappuccio FP, Miller MA, Lockley SW. Sleep, health and society: the contribution of epidemiology. Oxford: Oxford University Press; 2010.

25. Vedovato TG, Monteiro MI. Health Conditions and Factors Related to the Work Ability of Teachers. Ind Health. 2014;52(2):121-8.

26. Chellappa SL, Araújo JF. Confiabilidade e reprodutibilidade do questionário de hábitos do sono em pacientes depressivos ambulatoriais. Rev Psiquiatr Clin. 2007;34(5):210-4.

27. Chellappa SL, Araújo JF. O sono e os transtornos do sono na depressão. Rev Psiquiatr Clin. 2007;34(6):285-9.

28. Branco JC, Jansen K. Prevalência de sintomas osteomusculares em professores do ensino fundamental do maior colégio municipal da América Latina. Cien Cogn. 2011;16(3):109-15.

29. Dallepiane S, Bigolin SE. A presença de dor no cotidiano de professores da universidade regional do noroeste do estado do Rio Grande do Sul Unijuí. Rev Contexto Saude. 2004;3(7):231-9.

30. Cardoso JP, Araújo TM, Carvalho FM, Oliveira NF, Reis EJFB. Aspectos psicossociais do trabalho e dor musculoesquelética em professores. Cad Saude Publica. 2011;27(8);1498-506.

31. Silva FC, Sampaio RF, Mancini MC, Luz MT, Alcântara MA. A qualitative study of workers with chronic pain in Brazil and its social consequences. Occup Ther Int. 2011;18(2):85-95.

32. Mattos AIS, Araújo TM, Almeida MMG. Interação entre demanda-controle e suporte social na ocorrência de transtornos mentais comuns. Rev Saude Publica. 2017;51:48.

33. Caran VCS, Freitas FCT, Alves LA, Pedrão LJ, Robazzi MLCC. Riscos ocupacionais psicossociais e sua repercussão na saúde de docentes universitários. Rev Enferm UERJ. 2011;19(2):255-61.

34. Åkerstedt T. Psychosocial stress and impaired sleep. Scand J Work Environ Health. 2006;32(6): 493-501.

35. Cropley M, Dijk DJ, Stanley N. Job strain, work rumination, and sleep in school teachers. Eur J Work Organ Psychol. 2006;15(2):181-96.

36. Gao X, Ge H, Jiang Y, Lian Y, Zhang C, Liu J. Relationship between Job Stress and 5-HT2A Receptor Polymorphisms on Self-Reported Sleep Quality in Physicians in Urumqi (Xinjiang, China): A Cross-Sectional Study. Int J Environ Res Public Health. 2018;15(5):1034.

37. Kecklund G, Åkerstedt T. Apprehension of the subsequent working day is associated with a low amount of slow wave sleep. Biol Psychol. 2004;66(2):169-76.

38. Merey Z, Pişkin M, Boysan M, Şehribanoğlu S. Burnout among Turkish Teachers: The Influence of Sleep Quality and Job Satisfaction. H U J Educ. 2013;28(3):332-42.

39. Teixeira LN, Rodrigues AL, Silva FM, Silveira RCP. As possíveis alterações no estilo de vida e saúde dos professores. R Enferm Cent O Min. 2015;5(2):1669-83. 